\title{
SEMILATTICES AND A TERNARY OERATION IN MODULAR LATTICES
}

\author{
S. A. KISS
}

Before discussing the subject matter proper it is necessary to introduce the following: 1

LEMMA 1. The inequality
(1) $\{(x \cap(y \cap(v \cup z))\}$
$\cup(v \cap 8)$
$z) \subseteq v \cup(y \cap(x \cup z)) \cup(x \cap z)$

is identically satisfied in any lattice.

$$
\begin{aligned}
& \text { Proof. } x \cap y \cap\left(v \cup_{z}\right) \subseteq x \cap y \subseteq(x \cup z) \cap y \subseteq v \cup\left(y \cap\left(x \cup_{z}\right)\right) \text {, } \\
& v \cap z \subseteq v \subseteq v \cup(y \cap(x \cup z))
\end{aligned}
$$

and from these two inequalities follows

$$
\begin{aligned}
(x \cap y \cap(v \cup z)) \cup(v \cap z) & \subseteq v \cup(y \cap(x \cup z)) \\
& \subseteq v \cup(y \cap(x \cup z)) \cup(x \cap z) .
\end{aligned}
$$

For purposes of facility of expression the concept of semilattice is here introduced following Klein-Barmen [1]:2

Definition 1. A semilattice $L_{s}$ is a partially ordered system in which a relation $x \sigma y$ is defined which satisfies

$\mathrm{S} 1:$ For all $x, x \sigma x$,

S2: If $x \sigma y$ and $y \sigma x$, then $x=y$,

S3: If $x \sigma y$ and $y \sigma z$, then $x \sigma z$,

and in which any two elements $x$ and $y$ have a greatest lower bound or meet $x m y$.

It then follows that $x m y$ or any binary operation xoy which is closed, idempotent, commutative and associative defines, by means of the convention that $x \sigma y$ means $x m y=x$ or $x o y=x$, a semilattice $L_{s}$ in which $x m y$ or $x o y$ is the greatest lower bound of $x$ and $y$.

\section{Lemma 2. The ternary operation}

(2) $[x, t, y]=(x \cap(t \cup y)) \cup(t \cap y)=(x \cup(t \cap y)) \cap(t \cup y)$

on the elements of a modular lattice $L$ is closed and is an idempotent and 1948.

Received by the editors September 22, 1947, and, in revised form, January 24,

1 The author is indebted to Garrett Birkhoff for the proof of Lemma 1, and for helpful criticism.

${ }^{2}$ Numbers in brackets refer to the bibliography at the end of the paper. 
associative operation for a constant $t$. The expression $[x, t, y]$, for $t=$ const., is said to determine the "operational plane" $t$.

(3) The idempotent law

$$
[x, t, x]=x \text { for all } x \text { and } t
$$

holds because of the absorption law in $L$ :

$$
[x, t, x]=(x \cap(t \cup x)) \cup(t \cap x)=x \cup(t \cap x)=x .
$$

The proof of the associative law is somewhat longer, proceeding as follows:

Expanding the expression of the associative law

$$
[[x, t, y], t, z]=[x, t,[y, t, z]]
$$

one obtains

$$
\begin{aligned}
\{\{x \cap(t \cup y)) \cup(t \cap y)\} & \cap(t \cup z)\} \cup(t \cap z) \\
= & x \cap\{t \cup\{(y \cap(t \cup z)) \cup(t \cap z)\}\} \\
& \cup\{t \cap\{(y \cap(t \cup z)) \cup(t \cap z)\}\} .
\end{aligned}
$$

Some of the expressions on the right-hand side may be simplified by employing the absorption law and Dedekind's modular identity.

$$
\begin{aligned}
t \cup\{(y \cap(t \cup z)) \cup(t \cap z)\} & =t \cup(t \cap z) \cup(y \cap(t \cup z)) \\
& =t \cup(y \cap(t \cup z))=(t \cup y) \cap(t \cup z), \\
t \cap\{(y \cap(t \cup z)) \cup(t \cap z)\} & =t \cap(t \cup z) \cap(y \cup(t \cap z)) \mid \\
& =t \cap(y \cup(t \cap z))=(t \cap y) \cup(t \cap z) .
\end{aligned}
$$

Therefore,

$$
\begin{aligned}
\{\{(x \cap(t \cup y)) \cup(t \cap y)\} \cap(t \cup z)\} \cup(t \cap z) \\
=\{x \cap(t \cup y) \cap(t \cup z)\} \cup\{(t \cap y) \cup(t \cap z)\} .
\end{aligned}
$$

Putting $X=x \cap(t \cup y), Y=t \cup z, U=t \cap y, V=t \cap z$ where $V \subseteq Y$ and $U \subseteq t \subseteq Y$, the above formula becomes

$$
\{(X \cup Y) \cap Y\} \cup V=(X \cap Y) \cup(U \cap V)
$$

and, in view of $(X \cup U) \cap Y=(U \cup X) \cap Y=U \cup(X \cap Y)$,

$$
\{U \cup(X \cap Y)\} \cup V=(X \cap Y) \cup(U \cup V)
$$

which is an identity, thus concluding the proof.

An immediate consequence, then, is:

Theorem 1. The commutative "products" $[x, t, y]$ for a constant $t$, 
that is, those which satisfy $[x, t, y]=[y, t, x]$, form a semilattice in which the element $[x, t, y]$ is the greatest lower bound of $x$ and $y$.

THEOREM 2. Whether commutative or not, the "product" $[x, t, y]$ is always determined for a modular lattice $L$ and in the ternary operational system thus obtained any two operational planes $u$ and $v$ satisfy the following identical equation, namely,

$$
[[x, u,[y, v, z]], v,[y, u, z]]=[[x, v,[y, u, z]], u,[y, v, z]] .
$$

To prove this formula, it is expanded by means of (2) above and is subsequently shown to be an identity. Putting

$$
\begin{aligned}
& Y=u \cup(y \cap(v \cup z)) \cup(v \cap z), \\
& Z=u \cap((y \cap(v \cup z)) \cup(v \cap z)), \\
& U=v \cup(y \cap(u \cup z)) \cup(u \cap z), \\
& V=v \cap((y \cap(u \cup z)) \cup(u \cap z))
\end{aligned}
$$

where $Z \subseteq Y, V \subseteq U$ and, in view of Lemma $1, Z \subseteq U$ and $V \subseteq Y$, the equation (5) becomes

$$
(((x \cap Y) \cup Z) \cap U) \cup V=(((x \cap U) \cup V) \cap Y) \cup Z
$$

or, in view of Dedekind's modular identity,

$$
(V \cup(x \cap Y) \cup Z) \cap U=(Z \cup(x \cap U) \cup V) \cap Y .
$$

Since $Z \subseteq U$ and $V \subseteq U$ give $Z \cup V \subseteq U$ and, similarly, $Z \subseteq Y$ and $V \subseteq Y$ give $Z \cup V \subseteq Y$, the last equation becomes, in view of Dedekind's modular identity,

$$
Z \cup V \cup(x \cap Y \cap U)=Z \cup V \cup(x \cap U \cap Y) .
$$

$(5)$ is thus proven to be an identity.

EXAMPLE. Designating the elements of the nondistributive modular lattice $L_{5}$ by 0 (least), $a, b, c, e$ (greatest), the commutative "products" of the operational plane $[x, a, y]$ define a semilattice which is not a lattice, similar remarks applying to $[x, b, y]$ and $[x, c, y]$. The noncommutative products, namely, $[b, a, c]=b,[c, a, b]=c,[a, b, c]=a$, $[c, a, b]=c,[a, c, b]=a,[b, c, a]=b$ do not belong to the semilattices.

Being partially ordered systems, semilattices may be represented by diagrams. In $L_{5}$ links are preserved in all semilattices defined by (2) with constant $t$; it is the author's conjecture that this rule holds for the semilattices defined in any modular lattice.

When a lattice is distributive in addition to being modular, the expression (2) becomes 


$$
[x, t, y]=(x \cap t) \cup(t \cap y) \cup(y \cap x) .
$$

This is the ternary operation $(x, t, y)$ which was independently introduced by $\mathrm{Grau}^{3}[2]$ for Boolean algebras and by Birkhoff and Kiss [3] for distributive lattices in general.

It is obvious from the expression (2) of $[x, t, y]$ that $[x, t, y]$ $=[x, y, t] ;$ on the other hand the above example shows that, in some cases at least, $[x, t, y] \neq[y, t, x]$ and also $[x, t, y] \neq[t, x, y]$.

Complementation in distributive lattices has been defined by Birkhoff and Kiss [3] and can now be extended to modular lattices by means of the following:

Definition 2. The elements $x, x^{\prime}$ of a modular lattice $L$ are called "strictly complementary" if and only if

$$
\left[x, t, x^{\prime}\right]=t \text { for all } t \text {. }
$$

THEOREM 3. Strict complementation in a modular lattice is unique.

Proof. If $x$ has two complements, $x^{\prime}$ and $x^{\prime \prime}$, then $x^{\prime \prime}=\left[x, x^{\prime \prime}, x^{\prime}\right]$ $=\left[x, x^{\prime}, x^{\prime \prime}\right]=x^{\prime}$.

TheOREM 4. The 0 (least) and e (greatest) elements of a modular lattice are always strictly complementary; furthermore, the $[x, 0, y]$ and $[x, e, y]$ planes of the ternary lattice give the $x \cap y$ and $x \cup y$ operations, respectively.

$$
\begin{aligned}
& \text { Proof. [0,t,e] }=(0 \cap(t \cup e)) \cup(t \cap e)=0 \cup t=t, \\
& {[x, 0, y]=(x \cap(0 \cup y)) \cup(0 \cap y)=(x \cap y) \cup 0=x \cap y,} \\
& {[x, e, y]=(x \cap(e \cup y)) \cup(e \cap y)=(x \cap e) \cup y=x \cup y .}
\end{aligned}
$$

\section{BIBLIOGRAPHY}

1. F. Klein-Barmen, Axiomatische Untersuchungen zur Theorie der Halbverbande, Deutsche Mathematik vol. 4 (1939) pp. 32-43.

2. A. A. Grau, Ternary Boolean algebra, Bull. Amer. Math. Soc. vol. 58 (1947) pp. 567-572.

3. Garrett Birkhoff and S. A. Kiss, A ternary operation in distributive lattices, Bull. Amer. Math. Soc. vol. 58 (1947) pp. 749-752.

Standard Oil Development Company, New York, N. Y.

${ }^{3}$ Grau uses the notation $x^{2} y$ for $(x, t, y)$. 